\title{
Effects of Molecular Orientation and Crystallinity on Measurement by X-Ray Diffraction of the Crystal Lattice Modulus of Poly(vinyl alcohol) Prepared by Gelation/Crystallization from Solution
}

\author{
Masaru Matsuo, ${ }^{\dagger}$ Yuko Harashina, and Tetsuya Ogita* \\ Department of Clothing Science, Faculty of Home Economics, \\ Nara Women's University, Nara 630, Japan \\ * Department of Materials Science and Engineering, Faculty of Engineering, \\ Yamagata University, Yonezawa 992, Japan
}

(Received March 4, 1992)

\begin{abstract}
The crystal lattice modulus of poly(vinyl alcohol) was measured by X-ray diffraction using films which were prepared by gelation/crystallization from solution and elongated in a silicon oil after evaporating solvent. The measured crystal lattice modulus of specimen with Young's modulus $13-20 \mathrm{GPa}$ was in the range $200-220 \mathrm{GPa}$. These values were lower than that for polyethylene measured by using ultradrawn films. To check the morphology dependence of the measured crystal lattice modulus of poly(vinyl alcohol), a numerical calculation was carried out by considering molecular orientation and crystallinity. In this calculation, a three-dimensional model was employed, in which oriented crystalline layers are surrounded by oriented amorphous phase and the strains of the two phases at the boundary are identical. The theoretical results indicate that the difference between the crystal lattice modulus as measured by X-ray diffraction and the intrinsic value becomes less pronounced and Young's modulus of a sample becomes lower when a series coupling between crystalline and amorphous phases is predominant. A series of numerical calculations for the system with low crystallinity and orientational degree of amorphous chain segments indicates that the real value of the crystal lattice modulus is slightly higher than $200-220 \mathrm{GPa}$ measured by X-ray diffraction.

KEY WORDS Poly(vinyl alcohol) / Crystal Lattice Modulus / Numerical Calculation / X-Ray Diffraction / Series Coupling /
\end{abstract}

Since 1974, the preparation of polymeric fibers and films with high-strength and highmodulus has been extensively investigated for flexible polymers by gel-state spinning, ${ }^{1}$ ultradrawing of dried gel films ${ }^{2,3}$ ultradrawing of single-crystal mats, ${ }^{4}$ and two-step drawing of single-crystal mats. ${ }^{5}$ This research is based on the assumption that the theoretical Young's modulus and tensile strength of polymeric materials could be realized if the chains are fully aligned and if the specimen is almost completely crystalline. Results of interest have been obtained for polyethylene and polypropylene whose Young's moduli at $20^{\circ} \mathrm{C}$ are

* To whom all correspondence should be addressed. higher than $200 \mathrm{GPa}^{3,4}$ and $40 \mathrm{GPa},{ }^{6,7}$ respectively. These values are nearly equal to their crystal lattice moduli as measured by X-ray diffraction technique. Although the theoretical Young's modulus of polyethylene is one of the highest among crystalline polymers, the range of application is limited by its low melting point. Therefore, it is important to achieve ultradrawing of other crystalline polymers such as polyamide, polyester, and poly(vinyl alcohol) (PVA).

Among these polymers, PVA has been reported as a well-known polymer whose crystal lattice modulus measured by X-ray 
diffraction technique is slightly higher than for polyethylene. A question, however, arises as to whether or not the crystal lattice modulus measured by X-ray diffraction is equal to the intrinsic crystal modulus, if derived from measurements on a specimen with a low degree of molecular orientation and low crystallinity. PVA gels with molecular weight $<7 \times 10^{4}$ have the ability to form a film by evaporating the solvent, and interesting results have been reported by Watase et al. ${ }^{8,9}$ and Yamaura et al. ${ }^{10,11}$ For example, Watase et al. have reported that the modulus of the gels prepared by freezing/thawing for aqueous atactic PVA solution is higher than that of a gel prepared at temperatures above $0^{\circ} \mathrm{C}$ and increases by repeating the freezing/thawing cycle. Yamaura et al. ${ }^{11}$ studied the characteristics of dilute PVA solutions containing dimethyl sulfoxide $\left(\mathrm{Me}_{2} \mathrm{SO}\right)$ and water $\left(\mathrm{H}_{2} \mathrm{O}\right)$ and the properties of gels prepared from the solutions. They reported that turbidity of solution and the melting point of the resultant gels are dependent upon the composition of $\mathrm{Me}_{2} \mathrm{SO} / \mathrm{H}_{2} \mathrm{O}$. Hyon et al. ${ }^{12}$ produced high modulus PVA fibers by drawing of gels prepared by crystallization from semi-dilute solutions containing $70 \mathrm{vol} \% \mathrm{Me}_{2} \mathrm{SO}$, on the basis of a reported by Farrant ${ }^{13}$ that solvent mixtures ranging from 50 to $75 \mathrm{vol} \% \mathrm{Me}_{2} \mathrm{SO}$ do not freeze even at $-100^{\circ} \mathrm{C}$ because of hydrating. The resultant tensile strength and Young's modulus of the drawn fiber reached 2.8 and $80 \mathrm{GPa}$, respectively.

This paper deals with estimation of the crystal lattice modulus in the direction of the molecular chain axis by X-ray diffraction technique. PVA fibers prepared by the similar method prepared by Hyon et al. were used as specimens in order to check whether the stress within a specimen is everywhere the same as the external applied stress.

\section{EXPERIMENTAL}

PVA powder was used; the sample had polymerization of 17900 and a $98 \mathrm{~mol} \%$ hydrolysis. The $\mathrm{Me}_{2} \mathrm{SO} / \mathrm{H}_{2} \mathrm{O}$ composition was set to be $70 / 30$ to assure high drawability of PVA film based on reports by Hyon et al. ${ }^{12}$ The solutions were prepared by heating the well-blended polymer/solvent mixture at $105^{\circ} \mathrm{C}$ for $40 \mathrm{~min}$. The homogenized solution was poured into an aluminum tray kept at temperature $>100^{\circ} \mathrm{C}$ and then the tray was placed in a cold bath set at temperatures $<-100^{\circ} \mathrm{C}$, thus generating a gel. The gel kept at temperature $<-100^{\circ} \mathrm{C}$ for $5 \mathrm{~h}$ was immersed in a water bath at $20^{\circ} \mathrm{C}$ for a week to remove $\mathrm{Me}_{2} \mathrm{SO}$. The gel was dried to evaporate water under ambient conditions. When the content of water within a film was less than $500 \%$, the dry gel film was cut into strips of length $25 \mathrm{~mm}$ and width $10 \mathrm{~mm}$. The strip was clamped in a manual stretching device in such a way that the length to be drawn would be $10 \mathrm{~mm}$. The specimen was elongated manually at room temperature. After strethcing, the sample was fixed by the stretcher and dried to evaporate residual water for $2-3$ days from the dry gel under ambient conditions. The dried gel specimen was elongated to the maximum draw ratio (10 times) again manually in a silicon oil bath at $190^{\circ} \mathrm{C}$. Elongation in a silicon oil was done to avoid dehydration perfectly. Actually, slight dehydration was confirmed for elongation in a hot oven under nitrogen above $130^{\circ} \mathrm{C}$.

The density of the films was measured by pycnometry with $p$-xylene and carbon tetrachloride as the medium. Before the measurements were made, silicon oil on the film surface was removed by lens paper and then the specimen was cut into fragments. The fragments were immersed in ethanol to remove residual silicon oil in an ultrasonic washing instrument and subsequently vacuum-dried for 1 day. Crystallinity was calculated by assuming the densities of crystalline and amorphous phases to be 1.345 and $1.268 \mathrm{~g} \mathrm{~cm}^{-3}$, respectively. ${ }^{14}$ The density measurements to determine determine crystallinity were carried 
out several times to check the reproducibility of the values, since the difference of densities between crystalline and amorphous phases is not significant.

The crystal lattice modulus of the $(020)$ plane was observed by X-ray diffraction with a scintillation counter and a $12 \mathrm{~kW}$ rotatinganode X-ray generator (Rigaku RDA-rA operated at $200 \mathrm{~mA}$ and $40 \mathrm{kV}$ ). The X-ray beam was monochromatized with a curved graphite monochromator. Crystal strain was given by a constant-strethcing apparatus described elsewhere. ${ }^{15}$ The intensity distribution for the (020) plane was measured with point focusing using a system in which the incident beam was collimated by a circular collimator $2 \mathrm{~mm}$ in diameter, and the diffractioh beam was detected by a square slit with dimensions $0.9 \times 0.9 \mathrm{~mm}$. The specimen was mounted horizontally in the strethcing clamps of the apparatus. The intensity distribution was measured with a step-scanning device at a step interval of $0.1^{\circ}$, each at a fixed time of $40 \mathrm{~s}$, in the range $73-77^{\circ}$ (twice the Bragg angle).

\section{RESULTS AND DISCUSSION}

Figure 1 shows examples of the relationship between external applied stress and crystal lattice strain to know scattered values of the crystal lattice modulus of PVA due to differences of the samples used in the present experiment. The plots showed a linear relationship expect in the range of smaller lattice strain. This is probably due to non-uniform transmission of the drawing force within the specimen at initial elongation. It may be noted that the crystal lattice modulus ranges from 190 to $220 \mathrm{GPa}$ and the values for most of the specimens are $200-220 \mathrm{GPa}$. The corresponding Young's moduli of specimens were also estimated by the bulk strain measured simultaneously, which is listed in Table I. All values in Table I are slightly lower than

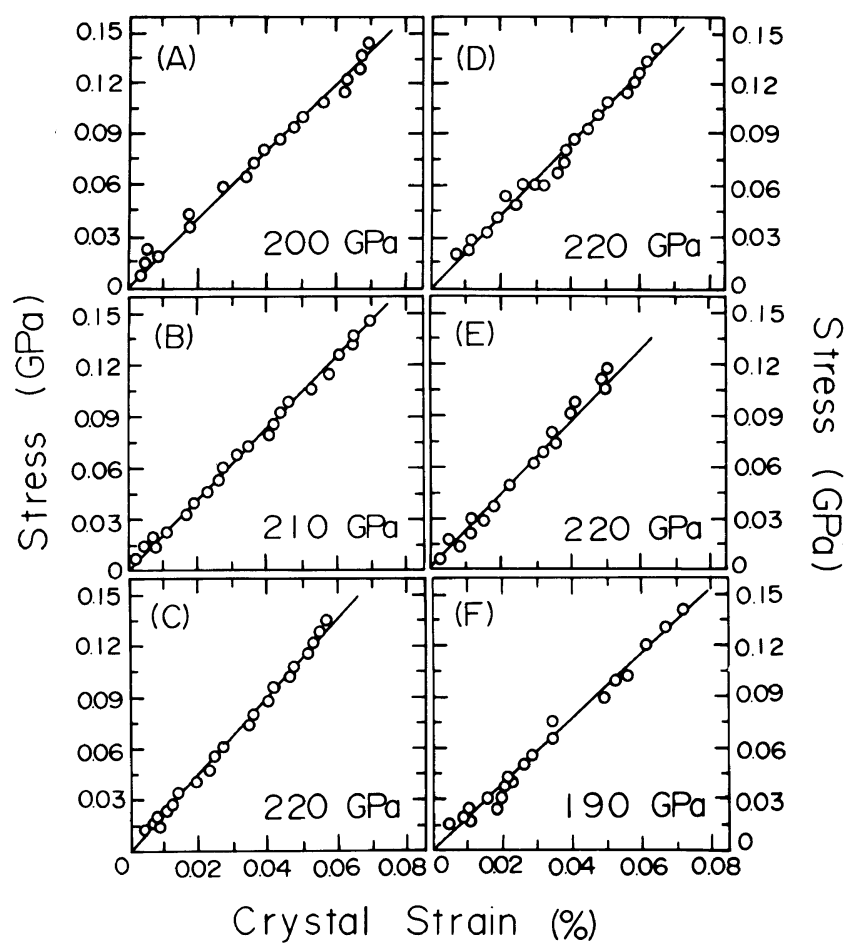

Figure 1. Stress-strain relationship for the (020) plane for drawn PVA films. 
Table I. Morphology and mechanical properties of drawn PVA films

\begin{tabular}{|c|c|c|c|c|}
\hline \multirow{2}{*}{ Specimen } & $\begin{array}{l}\text { Crystal lattice } \\
\text { modulus }\end{array}$ & $\begin{array}{l}\text { Young's } \\
\text { modulus }\end{array}$ & \multirow{2}{*}{$\begin{array}{c}\text { Crystallinity } \\
\%\end{array}$} & \multirow{2}{*}{$\begin{array}{l}\text { Second-order } \\
\text { orientation } \\
\text { of amorphous } \\
\text { chain segments }\end{array}$} \\
\hline & $\mathrm{GPa}$ & $\mathrm{GPa}$ & & \\
\hline A & 200 & 20.0 & 55 & 0.527 \\
\hline B & 210 & 7.5 & 55 & 0.0504 \\
\hline $\mathrm{C}$ & 220 & 13.5 & - & - \\
\hline $\mathrm{D}$ & 220 & 13.0 & - & - \\
\hline $\mathrm{E}$ & 220 & 15.0 & - & - \\
\hline $\mathrm{F}$ & 190 & 15.0 & - & - \\
\hline
\end{tabular}

reported by Sakurada et al. $(250 \mathrm{GPa})^{16}$ and Nakamae et al. $(235 \mathrm{GPa}) .{ }^{17}$ This discrepancy is probably due to the following two cases. The first is uncertainty in the linearization of the stress-lattice strain plots in the range of smaller lattice strain in this experiment and in the large lattice strain for the results of Sakurada et al. The second is variation of crystallinity and orientation of the $b$-axes (the crystal fiber axes). According to Sakurada's experiment, the Young's modulus of PVA fiber prepared by dry spinning was about $11 \mathrm{GPa}$, which is lower than for the specimens prepared from gels in this experiment except specimen B. This is due to poorer orientation of the $b$-axes of their specimen in comparison with the specimens used in the present study. As an example, the X-ray diffraction pattern (through view) from specimen $\mathrm{A}$ is shown in Figure 2. The diffraction spots indicate a high degree of crystallite orientation.

In order to verify the above two concepts, the crystal lattice modulus as measured by $\mathrm{X}$-ray diffraction and Young's modulus of a sample were calculated by considering the effects of orientation factors of crystal and amorphous chains and crystallinity. In the calculation, a previously introduced model was employed, ${ }^{18}$ in which oriented crystalline layers were surrounded by oriented amorphous phases and the strains of the two phases at the boundary are identical as shown in Figure 3. This model was first proposed by

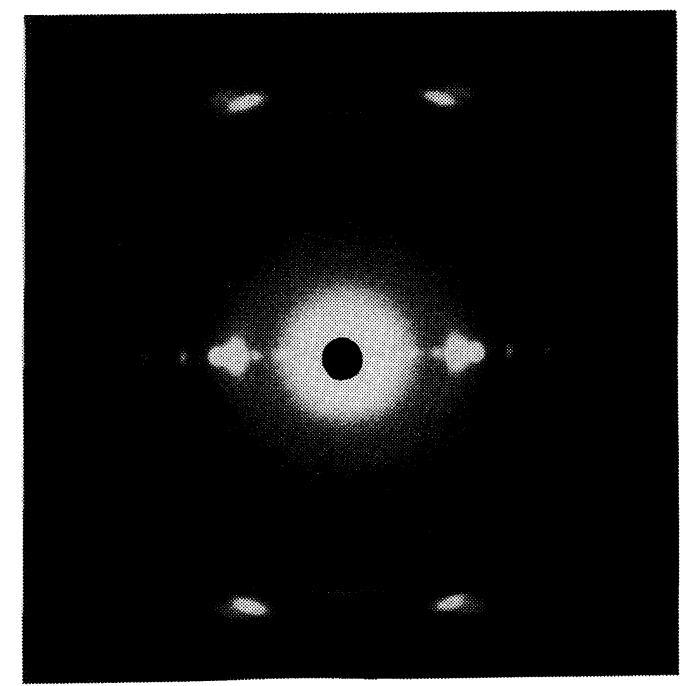

Figure 2. X-Ray diffraction pattern (through view) from drawn PVA film.

Hibi, ${ }^{19}$ which is thought to be a realistic representation of the morphology of crystalline polymers with a low degree of molecular orientation and low crystallinity. In this model, volume crystallinity $X_{\mathrm{c}}$ is represented by $\delta \mu^{2}$ using the fraction length $\delta$ and $\mu$ and external stress is applied in the $X_{3}$ direction.

The orientation factor $F_{l 0 n}(2 \leqq l \leqq 4,0 \leqq$ $n \leqq 4)$ and $G_{l 0 n}(2 \leqq l, n \leqq 4)$ of crystallites must be estimated from the orientation distribution functions of the reciprocal lattice vectors of crystal planes by the method of Roe and Krigbaum ${ }^{20,21}$; orientation factors $F_{l 00}^{\mathrm{am}}(2 \leqq l \leqq 4)$ of amorphous chain segments 


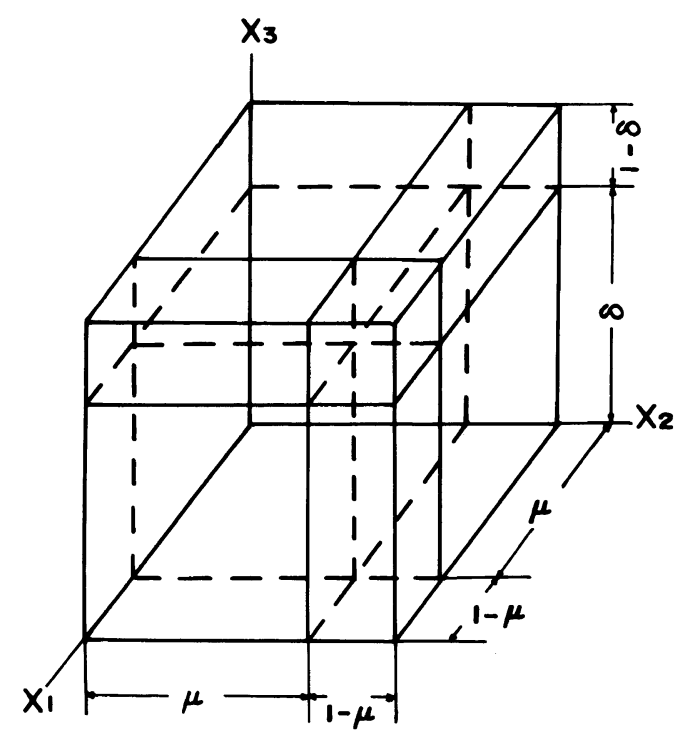

Figure 3. Composite structural unit of a semicrystalline polymer whose crystals are surrounded by the amorphous phase.

must be estimated by subtracting crystalline contributions from total birefringence. For the test specimens, the orientation distribution functions of the reciprocal lattice vectors of the crystal phases are too sharp to estimate orientation factors $F_{l 0 n}$ and $G_{l 0 n}$. Actually, $F_{200}$ and $F_{400}$ were almost unity and the other factors $F_{202}, F_{402}, F_{404}, G_{202}, G_{402}$, and $G_{404}$ were almost zero within experimental error. Thus, the above values are employed in the present paper.

The orientation of amorphous chain segments was estimated by subtraction of the crystal contribution from the total birefringence, assuming simple additivity proposed by Stein for biaxially oriented films. ${ }^{22}$ The values associated with biaxial orientational effect are scattered and this is not so significant. Thus, the second-order orientation factor of amorphous chain segments is calculated by assuming an uniaxial orientational mode. To obtain the above conclusion, the calculation was carried out by utilizing the values of bondpolarizabilities proposed by Clement, ${ }^{23}$ the crystal structure by Nitta et al., ${ }^{24}$ and the values of crystalline and amorphous densities as 1.345 and $1.269 \mathrm{~g} \mathrm{~cm}^{-3}$, respectively.

The parameters to calculate the secondorder orientation factors of the amorphous chain segments are obtained as follows:

crystalline indices

$$
\begin{aligned}
& n_{\mathrm{a}}=1.737 \text { ( } a \text {-axis) } \\
& n_{\mathrm{c}}=1.717 \text { ( } c \text {-axis) } \\
& n_{\mathrm{b}}=1.827 \text { ( } b \text {-axis, molecular axis) }
\end{aligned}
$$

amorphous indices

$$
\begin{gathered}
n_{\|}=1.750 \text { (parallel to molecular axis) } \\
n_{\perp}=1.662 \text { (perpendicular to molecular } \\
\text { axis) } \\
\left(n_{\|}-n_{\perp}\right)=0.088 \text { (intrinsic briefringence) }
\end{gathered}
$$

To calculate mechanical properties, the fourth-moment orientation factor of the amorphous chain segments must be estimated. Unfortunately, this moment cannot be obtained from birefringence measurements and therefore was estimated by assuming a common function. In this paper, if $\theta$ denotes the angle between an amorphous chain and stretching direction, the mean fourth-power of direction cosine $\left\langle\cos ^{4} \theta\right\rangle$ was calculated from an inversely superposed Gaussian type function $G(\theta)$ as follows ${ }^{25}$

$$
\left\langle\cos ^{4} \theta\right\rangle=\frac{\int_{0}^{\pi / 2} G(\theta) \cos ^{4} \theta \sin \theta \mathrm{d} \theta}{\int_{0}^{\pi / 2} G(\theta) \sin \theta \mathrm{d} \theta}
$$

where

$$
G(\theta)=\exp \left(-\theta^{2} / 2 \sigma^{2}\right)+\exp \left\{-(\theta-\pi)^{2} / 2 \sigma^{2}\right\}
$$

The values of $\left\langle\cos ^{4} \theta\right\rangle$ can be obtained graphically from the experimental values of $\left\langle\cos ^{2} \theta\right\rangle$ for amorphous chain segments, since the relation of $\left\langle\cos ^{2} \theta\right\rangle$ versus $\left\langle\cos ^{4} \theta\right\rangle$ can be calculated by varying the parameter $\sigma$. Thus, the fourth-order orientation factor $F_{400}^{\mathrm{am}}$ can 
be obtained.

The intrinsic elastic compliance of poly(vinyl alcohol) is given by Tashiro et al. ${ }^{26}$ using the $\mathbf{B}$ matrix in the normal coordinate treatments to save great amount of computer memory in the determination of the complicated crystal structure. They are given as follows:

$S_{i j}^{\mathrm{co}}\left(10^{-2} / \mathrm{GPa}\right)=$

$\left|\begin{array}{cccccc}6.87 & -3.98 & -0.03 & 0 & 0 & -5.04 \\ -3.98 & 10.90 & -0.08 & 0 & 0 & 0.73 \\ -0.03 & -0.08 & 0.35 & 0 & 0 & 0.07 \\ 0 & 0 & 0 & 9.28 & -0.68 & 0 \\ 0 & 0 & 0 & -0.68 & 61.05 & 0 \\ -5.04 & 0.73 & 0.07 & 0 & 0 & 27.59\end{array}\right|$

where $S_{33}^{\text {co }}$ and $S_{22}^{\text {co }}$ denote the elastic compliances of the crystal units in the directions of chain axis ( $b$-axis) and $c$-axis, respectively. From the value of $S_{33}^{\text {co }}$ in eq 3, the theoretical modulus is calculated to be $287.4 \mathrm{GPa}$. Calculation was carried out by using the crystal structure of Nitta et al. ${ }^{24}$ except for assuming the molecular chains of syndiotactic configuration instead of atactic configuration. This value is lower than for polyethylene $(313 \mathrm{GPa})$ calculated by Tashiro et al. $^{26}$ using the same method as in the case of PVA. According to their paper, ${ }^{26}$ the calculated value of the crystal lattice modulus of PVA along the chain direction without taking into account the intermolecular hydrogen bonding is 287.2 $\mathrm{GPa}$, almost the same as values considering hydrogen bonds. This indicates that the crystal lattice modulus in the chain direction is hardly affected by inter-chain bonding and is affected by the plane's area of a unit cell. If the calculations by Tashiro et al. are correct in terms of relative quantities (not absolute values), the crystal lattice modulus of PVA is lower than that of polyethylene. The ratio of $S_{33}^{\text {co }}$ of PVA and polyethylene becomes $0.916=(287 / 313)$ in the theoretical aspect. The ratio of the crystal lattice moduli as measured by X-ray diffraction becomes $0.889-0.978$ $(=200 / 225-220 / 225)$, which is in the range of the theoretical case. Here, two questions arise as to whether intra-chain hydrogen bonding causes significant effect on increase in the theoretical value of the crystal lattice modulus and whether the molecular chain of atactic configuration gives lower value of the crystal lattice modulus than that calculated for syndiotactic configuration. However, these questions still remain unsolved. Apart from the results by Sakurada et al. ${ }^{16}$ the values of crystal lattice modulus of PVA should be lower than that of polyethylene.

The elastic compliances $S_{\text {uu }}^{\text {ao }}$ of the amorphous phase needed in the calculation are not precisely known. Following our crude approximation discussed elsewhere, ${ }^{27}$ on the basis of the concept of Nomura et al., ${ }^{25}$ elastic compliances $S_{11}^{\text {ao }}$ and $S_{22}^{\text {ao }}$ were estimated by double differentiation of Lennard-Jones potential energy functions, while the compliance $S_{33}^{\text {ao }}$ was estimated assuming that the modulus along the chain axis is proportional to the number of molecular chains in the unit area perpendicular to the chain axis. Thus, we have

$$
\begin{aligned}
& S_{11}^{\mathrm{ao}}=S_{22}^{\mathrm{ao}}=\left(\rho_{\mathrm{c}} / \rho_{\mathrm{a}}\right)^{4} S_{11}^{\mathrm{c}^{\prime}} \\
& S_{33}^{\mathrm{ao}}=\left(\rho_{\mathrm{c}} / \rho_{\mathrm{a}}\right) S_{33}^{\mathrm{c}^{\prime}}
\end{aligned}
$$

where the compliances $S_{\mathrm{uv}}^{\mathbf{c}^{\prime}}$ corresponds to $S_{\mathrm{uv}}^{\mathrm{co}}$ at $\theta=0$ in the same notation in the previous paper.

$$
\begin{aligned}
& S_{11}^{\mathrm{c}^{\prime}}=\frac{1}{8}\left(3 S_{11}^{\mathrm{co}}+3 S_{22}^{\mathrm{co}}+2 S_{12}^{\mathrm{co}}+S_{66}^{\mathrm{co}}\right) \\
& S_{33}^{\mathrm{c}^{\prime}}=S_{33}^{\mathrm{co}}
\end{aligned}
$$

According to Hibi et al., ${ }^{19}$ the other compliances can be estimated as

$$
\begin{aligned}
& S_{44}^{\mathrm{ao}}=S_{55}^{\mathrm{ao}} \\
& S_{12}^{\mathrm{ao}}=-v_{21}^{\mathrm{ao}} S_{11}^{\mathrm{ao}} \\
& S_{23}^{\mathrm{ao}}=S_{13}^{\mathrm{ao}}=-v_{31}^{\mathrm{ao}} S_{33}^{\mathrm{ao}} \\
& 2 S_{11}^{\mathrm{ao}}=\left(2 S_{13}^{\mathrm{ao}}+S_{55}^{\mathrm{ao}}\right) \Omega \\
& 2 S_{11}^{\mathrm{ao}}=2 S_{12}^{\mathrm{ao}}+S_{66}^{\mathrm{ao}}
\end{aligned}
$$




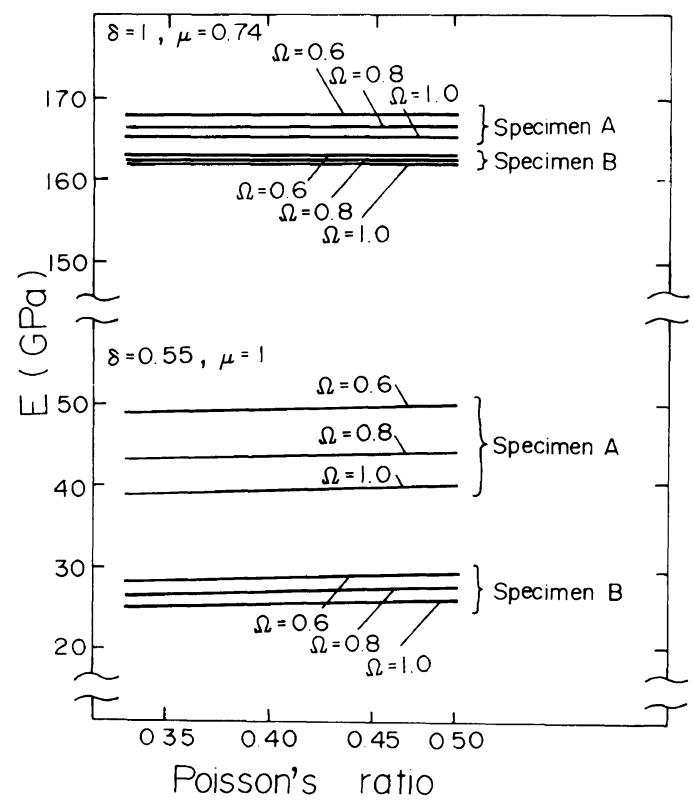

Figure 4. Poisson's ratio dependence of Young's modulus $E$ against $\Omega$ for drawn PVA films.

where $v_{21}^{\mathrm{ao}}$ and $v_{31}^{\mathrm{ao}}$ are Poisson's ratios of the amorphous phase and both values are assumed to be equal in actual calculation. The parameter $\Omega$ was first introduced by Hibi et $a l .{ }^{19}$ to calculate the elastic compliance $S_{55}^{\mathrm{ao}}$ of oriented PVA films. The value of $\Omega$ was in the range $0.7-1.0$ for the best fit between the experimental and calculated values of Young's moduli. In this paper, the value was set in the range $0.6-1.0$ to give wide variation of $S_{55}^{\text {ao }}$. The numerical calculation was carried out using specimens A and B listed in Table I, since the orientational degrees of amorphous chain segments are quite different from each other. For specimen A, the molecular orientation of the amorphous chain segments is predominant with respect to the strethcing direction, while for $\mathrm{B}$, the orientation is almost random.

Figure 4 shows the Poisson's ratio dependence of Young's modulus $E$ of a specimen against $\Omega$ and $\delta$ (or $\mu$ ) calculated for crystallinities by using the second- and fourth-order orientation factors of crystal and amorphous

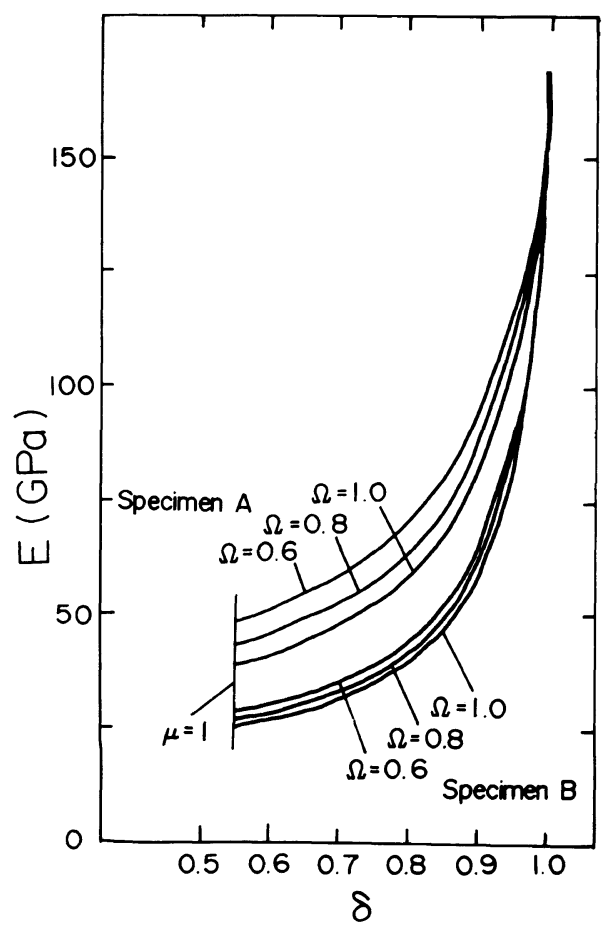

Figure 5. $\delta$-dependence of Young's modulus $E$ calculated for drawn PVA films.

phases of specimens A and B. As shown in this graph, the Young's modulus E depends on $\Omega$ and $\delta$ (or $\mu$ ) but is independent of the Poisson's ratio. That is, the numerical calculation indicates that Young's modulus calculated by the parameters for specimens $A$ and $\mathrm{B}$ becomes higher than $160 \mathrm{GPa}$ in spite of the considerable difference of the orientational degree of amorphous chain segments, when a parallel coupling model is employed $(\delta=1)$. In contrast, Young's modulus in the case of series model $(\mu=1)$ takes values lower than $50 \mathrm{GPa}$. Judging from the values between specimens $\mathrm{A}$ and $\mathrm{B}$, the calculated value of Young's modulus becomes lower with decreasing the orientational degree of amorphous chain segments. To check this tendency, the $\delta$-dependence of Young's modulus of a specimen was calculated.

Figure 5 shows the $\delta$-dependence of Young's modulus $E$ in bulk calculated for specimens A 


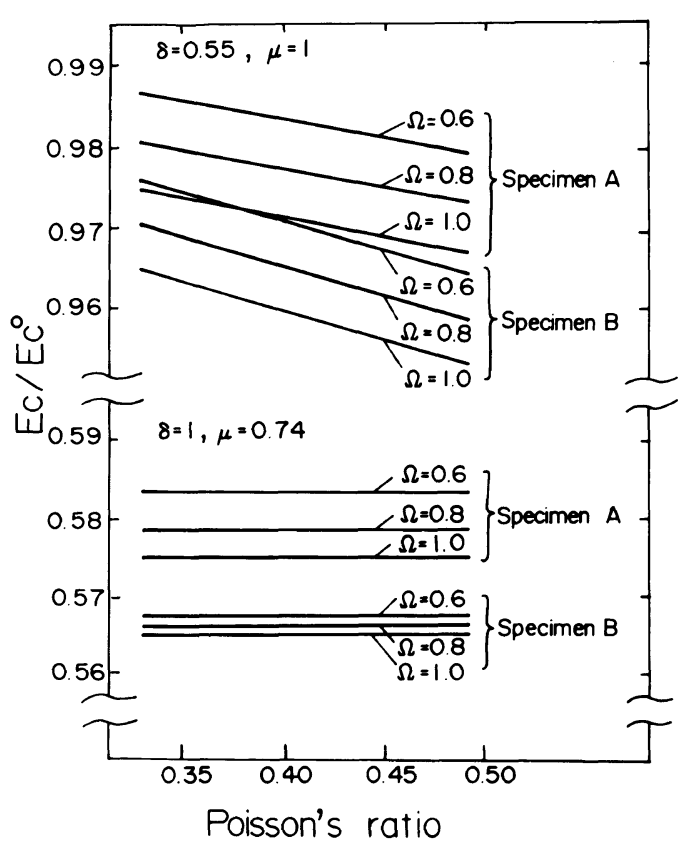

Figure 6. Poisson's ratio dependence of the ratio $E_{\mathrm{c}} / E_{\mathrm{c}}^{\mathrm{o}}$, in which $E_{\mathrm{c}}$ and $E_{\mathrm{c}}^{\mathrm{o}}$ correspond to the crystal lattice modulus measured by $\mathrm{X}$-ray diffraction and intrinsic crystal lattice modulus, respectively, for drawn PVA films.

and B. The Poisson's ratio is fixed at 0.39 . As shown in this figure, $E$ depends on $\Omega$ for specimen A but is almost independent for specimen $\mathrm{B}$ with almost random orientation of amorphous chain segments. At a constant value of $\Omega, E$ in increase with $\delta$ considerably. The results in Figures 4 and 5 predict that two specimens with the same crystallinity and degree of molecular orientation may have different Young's moduli, depending on the composite mode of crystalline and morphous phases. This tendency was already confirmed for cellulose. ${ }^{28}$

Figure 6 displays the Poisson's ratio dependence of the ratio $E_{\mathrm{c}} / E_{\mathrm{c}}^{\mathrm{o}}$, in which $E_{\mathrm{c}}$ and $E_{\mathrm{c}}^{\mathrm{o}}$ correspond to the crystal lattice modulus as measured by $\mathrm{X}$-ray diffraction and the intrinsic crystal lattice modulus of PVA, respectively. The value of $E_{\mathrm{c}}^{\mathrm{o}}$ was taken to be $287 \mathrm{GPa}$ calculated by Tashiro et al. The results in Figure 6 indicate that the values of
$E_{\mathrm{c}} / E_{\mathrm{c}}^{\mathrm{o}}$ are almost independent of the Poisson's ratio in a parallel model and the value calculated from a series coupling model decreases slightly with Poisson's ratio but as a whole, $E_{\mathrm{c}} / E_{\mathrm{c}}^{\mathrm{o}}$ is hardly affected by Poisson's ratio. This tendency is also observed for Young's modulus $E$ of a bulk specimen in Figure 4. The effect of $\Omega$ on $E_{\mathrm{c}} / E_{\mathrm{c}}^{\mathrm{o}}$ is not significant, indicating that average elastic compliance of the amorphous phase is hardly affected by $S_{55}^{\text {ao }}$ calculated from eq 6 . This tendency can be also recognized for Young's modulus of a bulk specimen in Figure 4. Thus it turned out that the shear modulus, $S_{55}^{\text {ao }}$, of the amorphous phase is not so effective in the determination of $E$ and $E_{\mathrm{c}} / E_{\mathrm{c}}^{\mathrm{o}}$. The values calculated for specimen A are slightly higher than those for specimen B but are essentially almost equal to those for specimen B. The slightly higher values for specimen A indicate that the orientational degree of amorphous chain segments causes slight increase in the value of $E_{\mathrm{c}} / E_{\mathrm{c}}^{\mathrm{o}}$. The significant effect on $E_{\mathrm{c}} / E_{\mathrm{c}}^{\mathrm{o}}$ is attributed to the composite mode of crystal and amorphous phase. The value of $E_{\mathrm{c}} / E_{\mathrm{c}}^{\mathrm{o}}$ is higher than 0.95 when a series model is employed $(\mu=1)$, while that is lower than 0.59 in the case of parallel model $(\delta=1)$.

Figure 7 shows the $\delta$-dependence of the ratio $E_{\mathrm{c}} / E_{\mathrm{c}}^{\mathrm{o}}$ for two different values of $\Omega$. $E_{\mathrm{c}} / E_{\mathrm{c}}^{\circ}$ decreases with increasing $\delta$ and is almost independent of $\delta$ and orientational degree of amorphous chain segments. Judging from the numerical results in Figure 7, the crystal lattice modulus as measured by X-ray diffraction is different from the intrinsic lattice modulus when parallel coupling between amorphous and crystalline phases is predominant, while the difference between both moduli becomes smaller as series coupling is predominant. Even in a series model $(\mu=1)$, however, the crystal lattice modulus as measured by X-ray diffraction is estimated to be below the intrinsic value.

In Table I, the crystal lattice modulus is mostly in the range $200-220 \mathrm{GPa}$ and the 


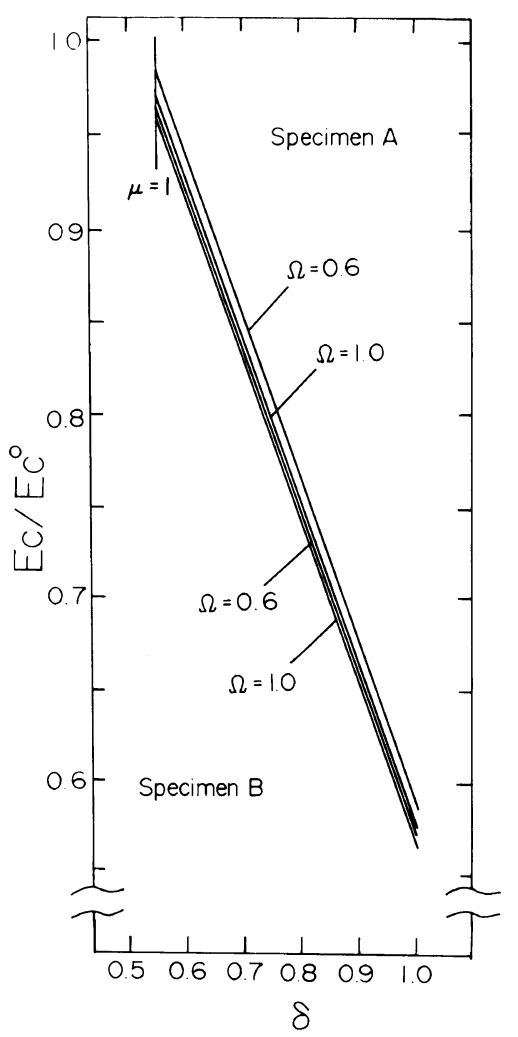

Figure 7. Ratio $E_{\mathrm{c}} / E_{\mathrm{c}}^{\mathrm{o}}$ versus $\delta$ calculated for drawn PVA films.

corresponding Young's moduli of specimens are mostly $13.5-15 \mathrm{GPa}$. This implies that the morphology of test specimens can be approximated as a series model because of low values of Young's modulus and almost constant values of the crystal lattice modulus measured by X-ray diffraction. If this is the case, the slight difference between the value as measured by X-ray diffraction and intrinsic value is attributed to the low orientational degree of amorphous chain segments and crystallinity. Thus, the real (intrinsic) values of PVA in the chain direction should be slightly higher than $200-220 \mathrm{GPa}$ measured by X-ray diffraction. Following the numerical calculation using the series coupling as shown in Figure 5, the maximum value of Young's modulus of a specimen was estimated as less than $50 \mathrm{GPa}$, in spite of the use of the intrinsic crystal lattice modulus, $287 \mathrm{GPa}$, by Tashiro et al. which is much higher than the observed values.

Here it should be noted that Young's modulus of drawn PVA films reported by several authors is higher than $110 \mathrm{GPa} .^{29}$ Surely, Young's modulus in bulk beyond $50 \mathrm{GPa}$ was also confirmed for specimens drawn in a hot oven beyond $150^{\circ} \mathrm{C}$ under nitrogen. ${ }^{30}$ This is probably due to increases in crystallinity and orientation of amorphous chain segments. In the experiment, however, the film was not obviously transparent but light brown by slight dehydration because of the existence of residual oxygen in a hot oven.

\section{CONCLUSIONS}

The crystal lattice modulus of PVA was measured by X-ray diffraction using drawn gel films with Young's moduli in the range $13-20 \mathrm{GPa}$. The values were mainly in the range $200-220 \mathrm{GPa}$. The crystal lattice modulus was slightly lower than that of polyethylene $^{15}$ and also lower than that of PVA reported by Sakurada et al. ${ }^{16}$ and Nakamura et al. ${ }^{17}$

Numerical calculations of the determination of the crystal lattice modulus by X-ray diffraction were carried out to investigate whether or not the homogeneous stress hypothesis is valid even in the test specimens with low crystallinity and low orientational degree of amorphous chain segments. The calculations were based on a model in which the oriented crystalline layers are surrounded by an anisotropic amorphous phases, and the strains of the two phases at boundaries are identical. The actual calculation was carried out using theoretical intrinsic elastic compliance by Tashiro et al. ${ }^{26}$ and by considering a system with perfect orientation of crystallites and orientation fluctuation of amorphous chain segments. The difference between the measured crystal lattice modulus and intrinsic 
value was less pronounced as series coupling between amorphous and crystalline phases was more predominant and consequently the measured modulus is close to the intrinsic value. Even if the morphology of the test specimens can be approximated as a series model, the measured value calculated by given parameters would be lower than the intrinsic value. This is probably due to the low crystallinity and orientation of amorphous chain segments. Thus, the real (intrinsic) values of PVA in the chain direction can be expected to be slightly higher than 200 $220 \mathrm{GPa}$ measured by X-ray diffraction. In comparison with the crystal lattice modulus of polyethylene, it turns out that the crystal lattice modulus in the chain direction is hardly affected by inter-chain hydrogen bonding and is affected by the cross section area of a unit cell perpendicular to the chain axis.

\section{REFERENCES}

1. P. Smith and P. J. Lemstra, J. Mater. Sci., 15, 505 (1980).

2. P. Smith, P. J. Lemstra, J. P. L. Pijpers, and A. M. Kiel, Colloid Polym. Sci., 259, 1070 (1980).

3. M. Matsuo, I. Inoue, and N. Abumiya, Sen- $i$ Gakkaishi, 36, 696 (1984).

4. K. Furuhata, T. Yokokawa, and K. Miyasaka, J. Polym. Sci., Polym. Phys. Ed., 22, 133 (1984).

5. T. Kanamoto, A. Tsuruta, K. Tanaka, and R. S. Porter, Polym. J., 15, 327 (1983).

6. M. Matsuo, C. Sawatari, and T. Nakano, Polym. J., 18, 759 (1986).

7. C. Sawatari and M. Matsuo, Macromolecules, 19, 2653 (1986).

8. M. Watase, K. Nishinari, and M. Nanbu, Polym.
Commum., 24, 52 (1987).

9. M. Watase and K. Nishinari, Macromol. Chem., 189, 871 (1988)

10. K. Yamaura, T. Karasawa, and S. Matsuzawa, J. Appl. Polym. Sci., 34, 2347 (1987).

11. K. Yamaura, T. Karasawa, and C. Matsuzawa, $J$. Appl. Polym. Sci., 37, 2709 (1989).

12. S. H. Hyon, W. I. Cha, and Y. Ikeda, Reports of Poval Committee, 89, 1 (1986).

13. J. Farrant, Nature, 205, 1284 (1965).

14. A. Keller, "Growth and Perfection of Crystals," D. Turnbell, Ed., John Wiley \& Sons, New York, 1958.

15. M. Matsuo and C. Sawatari, Macromolecules, 19, 2036 (1986).

16. I. Sakurada, Y. Nukushina, and T. Ito, J. Polym. Sci., 57, 651 (1962).

17. K. Nakamae, T. Nishino, and S. Takano, Kobunshi Ronbunshu, 43, 133 (1986).

18. M. Matsuo, Macromolecules, 23, 3261 (1990).

19. S. Hibi, M. Maeda, S. Makino, S. Nomura, and H. Kawai, Sen-i Gakkaishi, 29, 79 (1973).

20. R. J. Roe and W. R. Krigbaum, J. Chem. Phys., 40, 2608 (1964).

21. W. R. Krigbaum and R. J. Roe, J. Chem. Phys., 41, 737 (1964).

22. R. S. Stein, J. Polym. Sci., 24, 383 (1957).

23. C. Clement and P. Bothevel, C. R. Akad Sci. (Paris), 258, 4757 (1964).

24. I. Nitta, I. Taguchi, and Y. Chatani, Ann. Rept. Inst. Fiber Sci. Osaka Univ., 10, 1 (1957).

25. S. Nomura, S. Kawabata, H. Kawai, Y. Yamaguchi, A. Fukushima, and H. Takahara, J. Polym. Sci, A27, 325 (1969).

26. K. Tashiro, M. Kobayashi, and H. Tadokoro, Macromolecules, 11, 914 (1978).

27. C. Sawatari and M. Matsuo, Macromolecules, 19, 2726 (1986).

28. M. Matsuo, C. Sawatari, Y. Iwai, and F. Ozaki, Macromolecules, 23, 3266 (1990).

29. For example, T. Kunugi and T. Kawasumi, Preprint, IUPAC 32nd International Symposium on Macromolecules, 1988, p 431.

30. C. Sawatari, N. Yanagida, Y. Yamamoto, and M. Matsuo, Polymer, in press. 